\title{
Explore High Thermal Conductivity Amorphous Polymers using Reinforcement Learning
}

\author{
Ruimin $\mathrm{Ma}^{1, \perp, *}$, Hanfeng Zhang ${ }^{1, \perp}$, Tengfei Luo ${ }^{1,2, *}$
}

1. Department of Aerospace and Mechanical Engineering, University of Notre Dame, Notre Dame, Indiana 46556, United States

2. Department of Chemical and Biomolecular Engineering, University of Notre Dame, Notre Dame, Indiana 46556, United States

${ }^{\perp}$ These authors contribute equally

*Corresponding authors: rma4@nd.edu, tluo@,nd.edu

\begin{abstract}
Developing amorphous polymers with desirable thermal conductivity has significant implications, as they are ubiquitous in applications where thermal transport is critical. Conventional Edisonian approaches are slow and without guarantee of success in material development. In this work, using a reinforcement learning scheme, we design polymers with thermal conductivity above $0.4 \mathrm{~W} / \mathrm{m}$ $\mathrm{K}$. We leverage a machine learning model trained against 469 thermal conductivity data calculated from high-throughput molecular dynamics (MD) simulations as the surrogate for thermal conductivity prediction, and we use a recurrent neural network trained with around one million virtual polymer structures as a polymer generator. For all newly generated polymers with thermal conductivity $>0.400 \mathrm{~W} / \mathrm{m}-\mathrm{K}$, we have evaluated their synthesizability by calculating the synthesis accessibility score and validated the thermal conductivity of selected polymers using MD simulations. The best thermally conductive polymer designed has a MD-calculated thermal conductivity of $0.693 \mathrm{~W} / \mathrm{m}-\mathrm{K}$, which is also estimated to be easily synthesizable. Our demonstrated inverse design scheme based on reinforcement learning may advance polymer development with target properties, and the scheme can also be generalized to other materials development tasks for different applications.
\end{abstract}




\section{INTRODUCTION}

Machine intelligence, trained with a massive amount of data, has gained success in various fields, like playing Go,[1] predicting protein structures,[2] and recognizing images.[3] As more domainspecific data are accumulated,[4-7] advances have also been made for materials-by-design. Several works $[8,9]$ used reinforcement learning and recurrent neural network $(\mathrm{RNN})$ to generate material structures that satisfy certain properties, e.g., generating compounds active against the dopamine receptor type 2 . Some other works $[10,11]$ used variational autoencoder and Bayesian optimization for inverse material design, where the materials were projected onto a high-dimensional chemical space that could be tuned to generate materials with desirable properties. Research efforts have also been put forward to synthesize and experimentally validate these machine learning-designed materials. Mao et al. automated the design of architectural materials based on generative adversarial networks, successfully manufactured the designed architecture material, and experimentally validated their mechanical properties.[12] Wu et al. designed thermally conductive polymers based on Bayesian optimization and synthesized three polymers, whose thermal conductivity (TC) were then experimentally validated.[13] The inverse material design algorithms, enhanced by synthesizability estimation and property validation, promises to revolutionize the conventional Edisonian trial-and-error approach.

Designing amorphous polymers with high TC has been the interests of both industry and scientific research. Amorphous polymers are ubiquitous in thermal applications, which calls for the ability to design polymers with desirable TC.[14-16] However, the correlation between the polymer chemistry and TC is not well-established, which inspired academic interests in understanding heat transfer physics in polymers and its relation with polymer chemistry.[14, 17] While certain 
underlying physics, such as that stiffer chain backbone,[18, 19] stronger polar groups,[20-23] and denser linker molecules, [24] could lead to higher TC, has been revealed, formulating a model that can accurately describe the polymer chemistry-TC relation is still out of reach due to the complexity of such a relationship. Without this quantitative relationship established, developing polymers with target TC needs to rely on limited and incomplete physics understanding, which is slow and without guarantee of success.

Data-driven approaches can potentially establish such relationships using machine learning, but such efforts have been limited for polymer TC, mainly due to the lack of data. Wu et al. implemented a transfer learning scheme to mitigate the small data problem and developed a surrogate model based on merely 28 experimental data, which was further used for Bayesian molecular design.[13] Even though the quantitative structure-TC relationship was dramatically improved with transfer learning,[25] the information about which polymer structures tend to have high TC was still limited since the data contained only five polymer TC values above $0.300 \mathrm{~W} / \mathrm{m}$ $\mathrm{K}$, causing the designed polymers to be lack of diversity.

High-throughput simulation data are playing more important roles in materials design, by taking advantage of the increasingly affordable and fast computing infrastructure. There are a number of studies on designing materials with machine learning and simulation data, such as finding molecules with desirable thermodynamic properties based on high-throughput force field simulation,[26] exploring high-temperature polymers based on high-fidelity molecular dynamics (MD) simulations,[27] exploring polymer dielectric properties using density functional theory (DFT) calculations, $[28,29]$ screening intermetallic alloys with certain surface properties based on 
high-throughput DFT calculations,[30] and designing architecture materials with specific mechanical properties based on high-throughput finite element simulations.[12] Recently, Ma et al. calculated TC for 469 amorphous polymers based on high-throughput MD simulations, where 35 polymer structures from seven polymer classes were found to have TC above $0.400 \mathrm{~W} / \mathrm{m}-\mathrm{K}$, offering more information on the polymer chemistry-TC relationship in the high TC region.[31] Some of the MD-labeled TC were also validated by available experimental data, which can be potentially leveraged for inverse design of amorphous polymers with high TC.

In this work, we employ an inverse material design algorithm[9] based on reinforcement learning to explore amorphous polymers with high $\mathrm{TC}(>0.400 \mathrm{~W} / \mathrm{m}-\mathrm{K})$ by leveraging the $469 \mathrm{MD}$ simulation data generated in ref. [31]. A machine learning-generated benchmark database, PI1M (a.k.a., 1 million polymers for Polymer Informatics),[32] which contains $\sim 1$ million virtual polymer structures, is employed for training the polymer generator based on a RNN, using which new polymer structures can be generated. A random forests (RF) model is trained to quantify the chemistry-TC relationship on the 469 MD simulation data, which provides feedback on (i.e., predicts TC for) newly generated polymers. The inverse polymer design process is discussed in detail via hyperparameter study and based on statistical analysis of generated polymers. The designed polymers have predicted TC between 0.400 and $0.430 \mathrm{~W} / \mathrm{m}-\mathrm{K}$, whose synthetic accessibility and chemical diversity are characterized and leveraged for selecting candidates for further MD validation. We finally validate 30 polymer structures via MD simulations, whose TC range from $0.360 \mathrm{~W} / \mathrm{m}-\mathrm{K}$ to $0.693 \mathrm{~W} / \mathrm{m}-\mathrm{K}$, which are chemically diversified and estimated to be easily synthesizable based on the calculated synthetic accessibility score. 


\section{METHODS}

Dataset. The PI1M database,[32] which contains 995,799 machine learning-generated virtual polymer structures, is used to train the polymer generator that can generate new polymers. PI1M is a machine-learned benchmark database based on the PolyInfo database[5], where $\sim 12,000$ homo-polymer structures were manually collected from the PolyInfo database and then used to train a RNN generative model. 995,799 polymer structures were sampled from this generative model to form PI1M. The polymers are represented using their corresponding monomer structures with polymerization information indicated, which are further stored in the SMILES[33] format (Figure 1a). These SMILES are referred to as p-SMILES,[32] i.e., SMILES for polymers. It differs from the SMILES for drug-like molecules since it provides polymerization points as “*” in the string (Figure 1a). The p-SMILES represents the polymer structures as sequences of characters, including the information of atoms, rings, and branches. The p-SMILES used in this work are limited to homopolymers,[34] which consists of only one type of monomer and two polymerization points, but the same strategy may be extend to heteropolymer built on two or more types of monomers in future when relevant data are accumulated. We collect the MD-calculated TC data for 469 amorphous polymers from ref. [31], which are used to train a RF model to describe the relationship between p-SMILES and TC. The TC distribution of these 469 amorphous polymers is shown in Figure 1b. The p-SMILES, which represents the polymer structures, are used as the inputs for all the implementations below. 


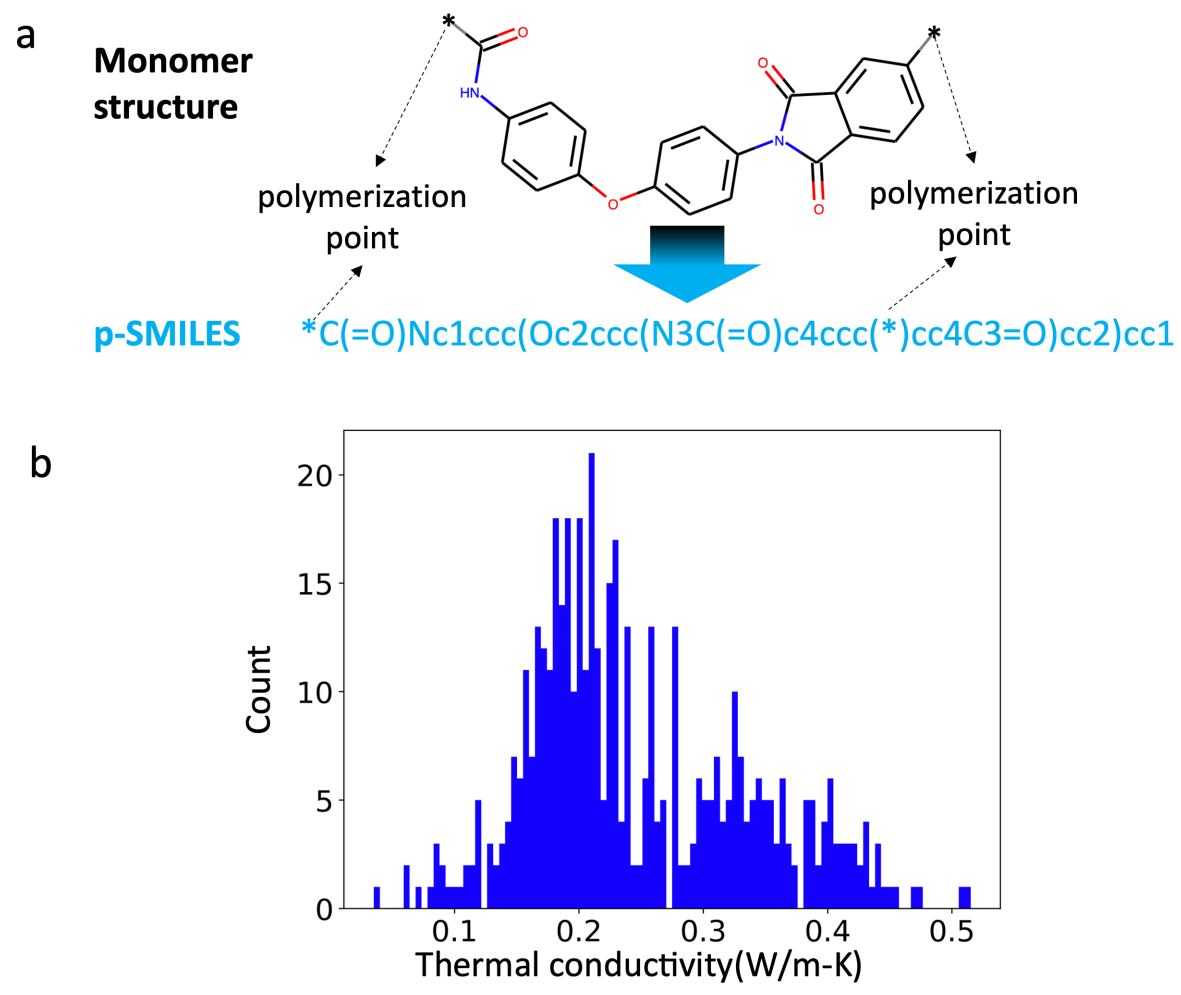

Figure 1. (a) An example of the representation of polymer structure, p-SMILES, used in this study; (b) TC distribution of 469 polymer structures collected from ref.[31], which was calculated using high-throughput MD simulations.

Regressor. Generating materials with desirable properties relies on the feedback from properties of the generated materials that can be either directly calculated (e.g., molecular weight[35]) or estimated by high-fidelity surrogate models. $[9,13] \mathrm{TC}$ is a property that usually takes weeks or months to experimentally measure or days to calculate in MD simulations. Thus, for efficient TC evaluation, we choose to establish a regressor to quantify the p-SMILES-TC relation, which is leveraged to instantaneously predict TC for (i.e., give feedback on) the newly generated polymers. We first numerically represent the 469 p-SMILES using polymer embedding,[32] which is a machine learning representation trained based on PI1M to converts the p-SMILES into continuousvalued vectors with 300 in dimension. More details of the polymer embedding representation can 
be found in Ref. [32]. A RF model[36] is then trained to quantify the polymer embedding-TC relation. The number of trees in the RF model is set to 100 , which is determined from hyperparameter tuning. The predictive accuracy of the RF model is evaluated by $R^{2}$ and measured in 10-fold cross-validation. Based on the results in Figure 2, the RF can achieve relatively high predictive accuracy $\left(R^{2}=0.828\right)$, and to make the best of the information embedded in the data, it is trained on all 469 data to predict TC for the generated p-SMILES in the inverse polymer design process. Scikit-learn[37] is used for the RF implementation.

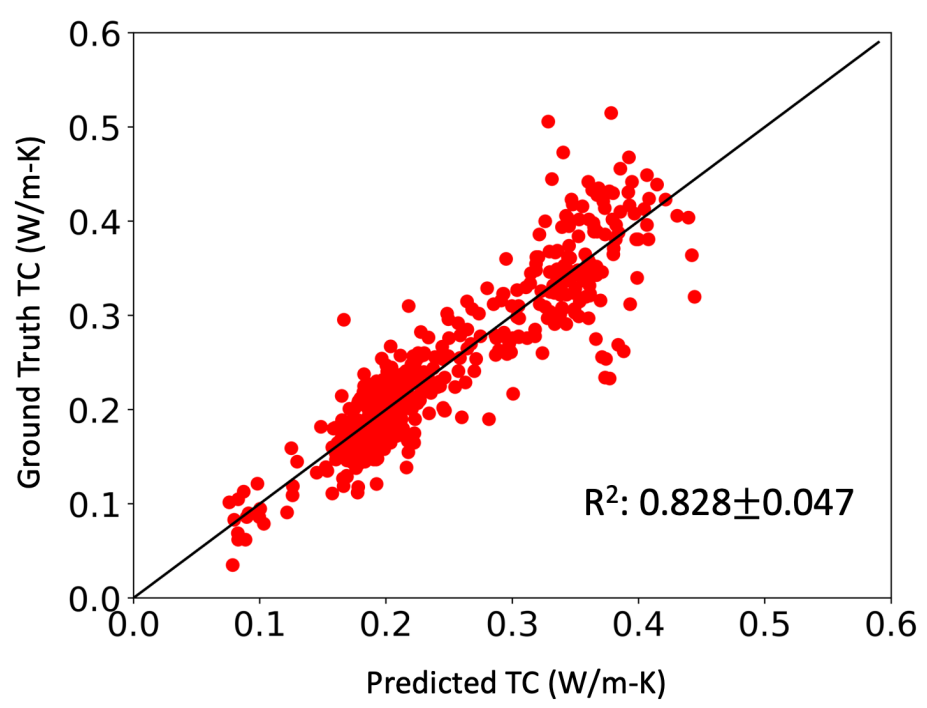

Figure 2. The pair plot between RF predictions and ground truth (MD-calculated TC) on all validation sets in the 10-fold cross-validation. Predictive accuracy is evaluated by $R^{2}$ and measured on the validation sets in the 10 -fold cross-validation.

Polymer generator. The p-SMILES, as can be seen from Figure 1a, are sequential data in nature. $\mathrm{RNN},[38]$ a neural network architecture that is well-suited for sequential data modelling, is employed to learn the p-SMILES in PI1M and serves as the polymer generator after training. The p-SMILES in PI1M are tokenized into sequences of characters $\left(X_{1}, X_{2}, \ldots, X_{t-1}, X_{t}, \ldots, X_{N}\right)$, where 
two additional tokens of "GO" and "EOS" are added to initialize and terminate a p-SMILES generation. For example, the p-SMILES “* $\mathrm{OC}(\mathrm{CC}) \mathrm{O}$ *” is tokenized into ("GO", “*”, "O", "C", “(”, “C”, “C”, “)”, “O”, “*”, “EOS”). At each step $t$, RNN learns the probabilistic distribution $P\left(X_{t+1} \mid X_{1}, X_{2}, \ldots, X_{t}\right)$, which describes the conditional probability of generating the new character given those before it. The training aims to maximize the likelihood assigned to the correct character, and the parameters of RNN are updated by back-propagation through steps.[39] New p-SMILES can then be generated from the trained RNN by sampling tokens from the trained conditional probabilities. In the generation process, the first input character ("GO") is given to initialize the sampling, and at each step, an output character is sampled from the trained RNN, which is used as the next input. When "EOS" is sampled, the p-SMILES is considered completed. The RNN employed consists of three layers of 512 gated recurrent units (GRU).[40] The size of character embedding (i.e., numerical representation of characters in p-SMILES) at the input layer is 128. The sequence length used in RNN is monitored by the maximum length of the p-SMILES used in each training batch, i.e., when doing the batch training, the p-SMILES with varying length are padded into the same length before feeding into RNN (usually padded with vectors of value zero, e.g., $[0,0, \ldots, 0]$, which poses no impacts on training), which is determined by the maximum length of the p-SMILES used in one batch. The RNN is trained via gradient descent with a batch size of 128 and the Adam[41] optimizer, and the learning rate is initialized at 0.001 and decays by a factor of 0.030 every 30 iterations. PyTorch[42] is used for the RNN implementation.

Inverse polymer design. We employ a reinforcement learning algorithm similar to that deployed for designing drug-like molecules.[9] In our study, two polymer generators, modelled by two RNNs respectively, are employed (Figure 3), whose architecture information and implementation 
details are mentioned in the Polymer generator section. The first polymer generator is trained on PI1M to learn the training data distribution, and we call it the baseline polymer generator $\left(P G_{\text {baseline }}\right)$. The second polymer generator is trained to generate p-SMIILES with desirable TC, and we call it polymer generator for TC $\left(P G_{T C}\right) . P G_{T C}$ is initialized by copying the weights of $P G_{\text {baseline. }}$ In each training step, batches of p-SMILES are sampled from $P G_{T C}$, whose TC are then predicted by the RF model trained above (details can be found in the Regressor section). The sampled p-SMILES $(P)$ and their corresponding predicted TC $(T)$ are then used to train $P G_{T C .} P$ is first passed through $P G_{T C}$ and $P G_{\text {baseline }}$ respectively to obtain their corresponding log likelihood $\log \operatorname{Prob}(P)_{T C}$ and $\log \operatorname{Prob}(P)_{\text {baseline }}$, calculated by $\sum_{t=1}^{t=N-1} \log \operatorname{Prob}\left(X_{t+1} \mid X_{1}, \ldots, X_{t}\right)$ (note $P$ is decomposed into sequences of characters $\left(X_{i}\right)$ in RNN as described in the Polymer generator section), which are both negative continuous-valued numbers. $T$ is used for building the score function ( $S$ ) (if $T>=$ threshold, then $S=T$; else, $S=0$ ), where the threshold is a TC value (e.g., 0.400 if we intend to generate $\mathrm{p}$-SMILES with $\mathrm{TC}>=0.400 \mathrm{~W} / \mathrm{m}-\mathrm{K})$. The loss function for training $P G_{T C}$ is defined as $\mathcal{J}=\left(\log \operatorname{Prob}(P)_{T C}-\left(\log \operatorname{Prob}(P)_{\text {baseline }}+\alpha S\right)\right)^{2}$, where $\alpha$ is a hyperparameter that can be tuned. The loss function is minimized by the Adam optimizer, with the same learning rules specified above in the Polymer generator section. The training aims to increase the sampling likelihood of p-SMILES with TC $>=$ the threshold. We do not alter the weights of $P G_{\text {baseline }}$ in the inverse polymer design process, since during the training, we would like $P G_{T C}$ to be anchored to $P G_{\text {baseline, }}$ which has learned both the syntax and distribution of p-SMIELS in PI1M.[9] In this way, the generated p-SMILES from $P G_{T C}$ is more likely to correspond to valid polymers. 


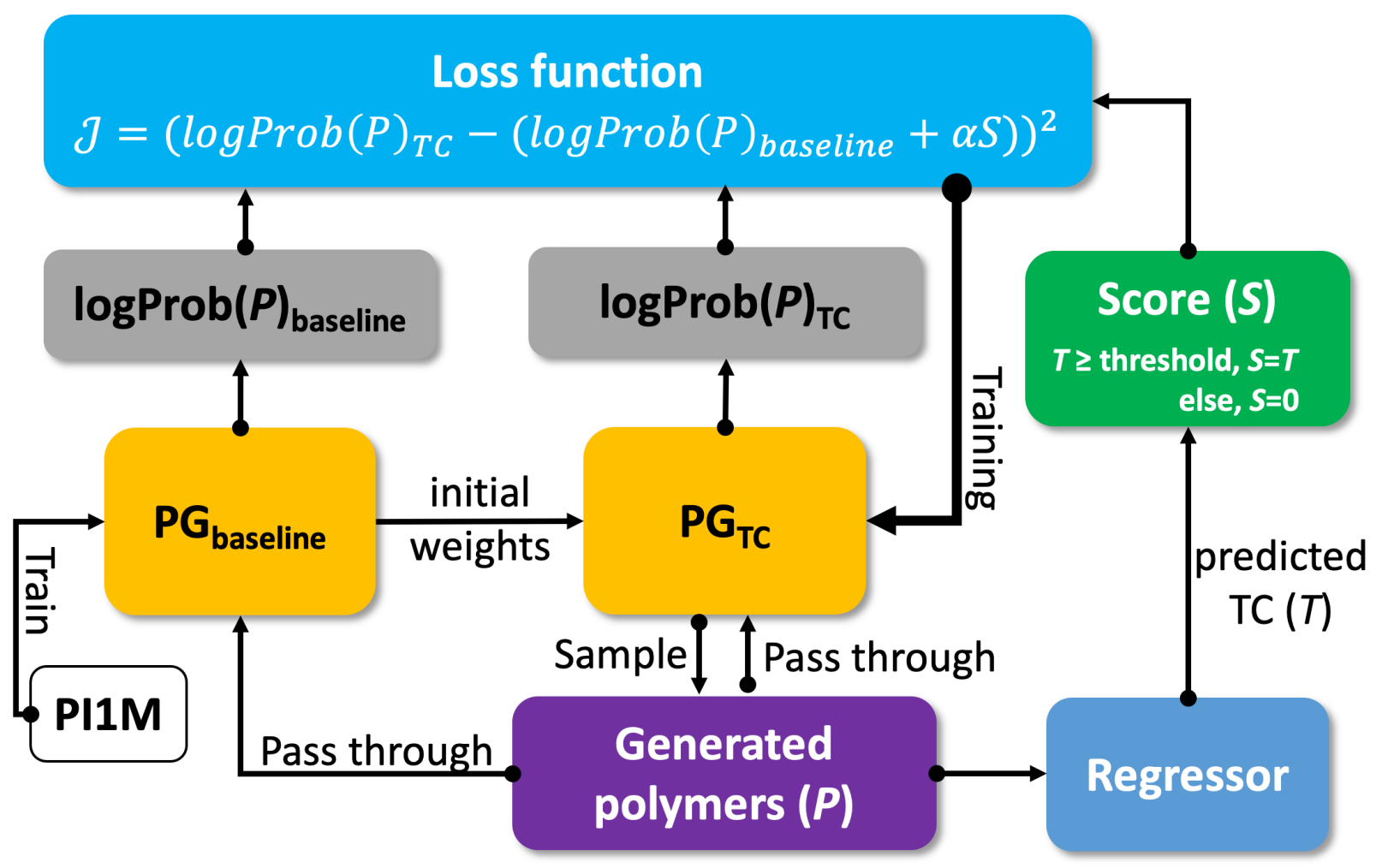

Figure 3. The schematic of the reinforcement learning algorithm for polymer design with target threshold of TC.

\section{RESULTS}

We first train the $P G_{\text {baseline }}$ on PI1M, which is divided into batches, with 256 p-SMILES in each batch. After training $P G_{\text {baseline }}$ for every 30 batches (30 batches $=1$ iteration), 128 p-SMILES are sampled from it, whose validities are evaluated by the three down-selection criteria (i.e., RDKit[43] chemical validation; number of "** $=2$; number of bonds to "** $=1$ ) originally used to build PI1M.[32] The motivation is to reinforce the generated p-SMILES to be similar to the p-SMILES used for training to increase the validity. The valid ratio of generated p-SMILES is leveraged to judge whether $P G_{\text {baseline }}$ has had sufficient training. Based on the results in Figure 4a, the valid ratio converges to $\sim 90 \%$ in less than 250 iterations, indicating $P G_{\text {baseline }}$ as an effective polymer 
generator. We have also examined the chemical space coverage of the $P G_{\text {baseline }}$-generated pSMILES and compared it to that of PI1M using t-SNE.[44] As can be seen in the t-SNE plot of the polymer representations (Figure $4 \mathbf{b}$ ), the two coverages in general overlap, suggesting that $P G_{\text {baseline }}$ can generate $\mathrm{p}$-SMILES with a distribution similar to that of the benchmark data in PI1M. The highlighted part at the right corner shows an example of how the trained $P G_{\text {baseline }}$ can generate polymers around existing ones with some extrapolation capability.

a

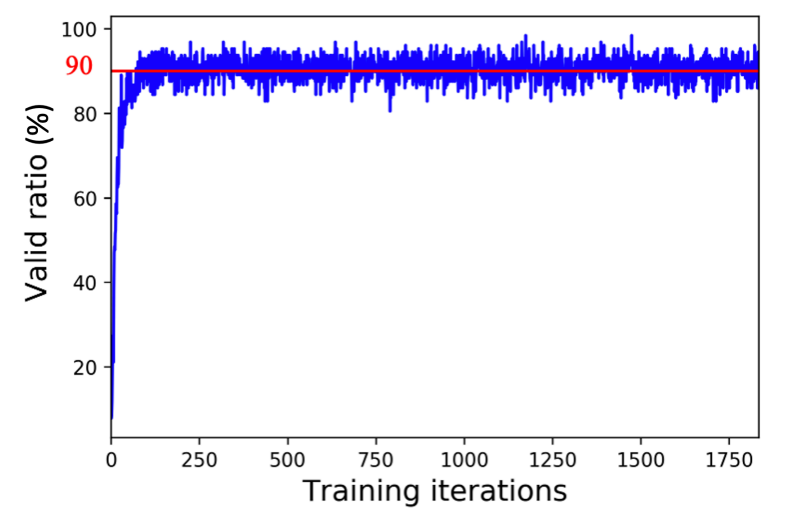

b

$$
\begin{aligned}
& \text { PIIM p-SMILES } \\
& \text { generated p-SMILES }
\end{aligned}
$$
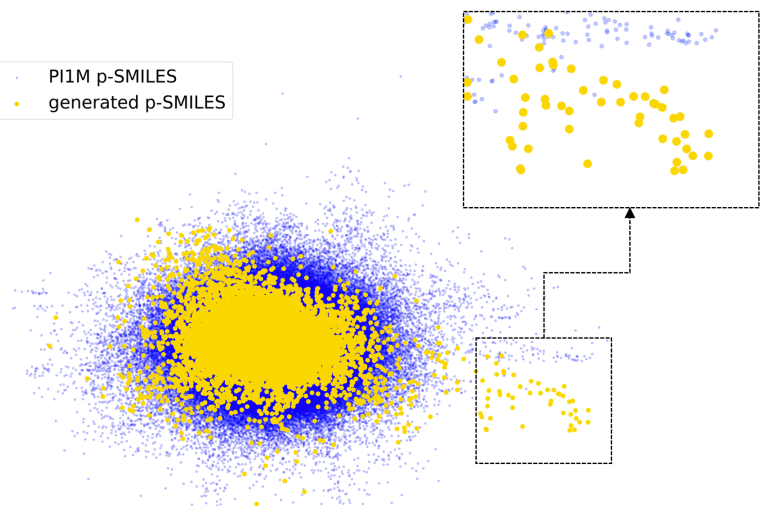

Figure 4. (a) Valid ratio of generated p-SMILES as a function of training iterations; (b) Chemical space coverages of both generated p-SMILES by $P G_{\text {baseline }}$ and p-SMILES from PI1M visualized using t-SNE, where 10,000 generated p-SMILES by $P G_{\text {baseline }}$ and 995,799 p-SMILES from PI1M are first represented by polymer embedding and then projected onto the 2-D chemical space for visualization via t-SNE.

After obtaining a well-trained $P G_{\text {baseline, }}$ we start to design p-SMILES with higher TC using the inverse polymer design algorithm mentioned above. The threshold in the score function $(S)$ is set to $0.400 \mathrm{~W} / \mathrm{m}-\mathrm{K}$, a TC value that is relatively high, but not common, for amorphous polymers. For example, in the PolyInfo database, the TC ranges from 0.100 to $0.438 \mathrm{~W} / \mathrm{m}-\mathrm{K}$, with 2 out of 70 available TC data having values above $0.400 \mathrm{~W} / \mathrm{m}-\mathrm{K}$. Among the $469 \mathrm{MD}$-calculated TC for our 
regressor training, only 35 of them are above $0.400 \mathrm{~W} / \mathrm{m}-\mathrm{K}$. The $P G_{T C}$ is initialized by copying the weights of $P G_{\text {baseline }}$ and trained for 1,000 steps. In each step, 64 p-SMILES are sampled from $P G_{T C}$, whose validities are also checked by the three criteria mentioned previously. Only the valid p-SMILES with predicted TC $>=0.400 \mathrm{~W} / \mathrm{m}-\mathrm{K}$ are employed to build the cost function, which, in turn, is used to train the $P G_{T C}$. Using p-SMILES sampled from the $P G_{T C}$ to train itself will gradually skew the distribution of the generated polymers so that more of them have TC above the threshold of $0.400 \mathrm{~W} / \mathrm{m}-\mathrm{K}$. When p-SMILES with higher TC are sampled as training goes on, the $P G_{T C}$ learns to sample the high TC polymers with higher probability.[45]

As mentioned in the Inverse polymer design section, the hyperparameter $\alpha$ can impact the learning process, thus leading to different generated p-SMILES. We train $P G_{T C}$ with different $\alpha$ values, and for each $\alpha$ value, the $P G_{T C}$ is trained for 1,000 steps. 1,000 p-SMILES are sampled from the $P G_{T C}$ every 100 training steps, and we perform statistical analysis on the total 10,000 generated p-SMILES with different evaluation metrics, aiming to find an appropriate hyperparameter $\alpha$. The results of such a hyperparameter study are shown in Table 1. Too small a $\alpha$ value (e.g., 10 or 30) would lead to inefficient learning, indicated by a small number of generated p-SMILES with predicted TC $>=0.400 \mathrm{~W} / \mathrm{m}-\mathrm{K}$, which in turn, could prevent the $P G_{T C}$ sufficiently exploring p-SMILES with high TC. As $\alpha$ value becomes larger (e.g., 100), the number of generated p-SMILES with TC $>=0.400 \mathrm{~W} / \mathrm{m}-\mathrm{K}$ increases significantly. However, when $\alpha$ value is excessively large (e.g., 500), the number of generated p-SMILES with predicted TC $>=0.400$ $\mathrm{W} / \mathrm{m}-\mathrm{K}$ decreases significantly. The $P G_{T C}$ deviates from the $P G_{b a s e l i n e}$ greedily to maximize its expectation (details discussed in Inverse polymer design section) if $\alpha$ is too large, thus leading to a large portion of generated p-SMILES that are unreasonably different from the p-SMIELS used 
for training, which cannot pass the down-selection validation criteria mentioned above, indicated by a low valid ratio in Table 1. Based on these results, we set $\alpha$ to 100 for the rest of the study.

Table 1. The impact of hyperparameter $\alpha$ on the inverse polymer design.

\begin{tabular}{cccc}
\hline & Highest predicted & Number of & \\
& TC of generated $\mathrm{p}-$ & generated $\mathrm{p}-$ & \\
SMILES (W/m-K) & SMILES with & \\
& & TC $>0.40 \mathrm{~W} / \mathrm{m}-\mathrm{K}$ & \\
\hline 10 & 0.410 & 7 & 0.937 \\
30 & 0.420 & 51 & 0.936 \\
100 & 0.430 & 7,713 & 0.918 \\
500 & 0.428 & 2,496 & 0.297 \\
\hline
\end{tabular}

We further analyze the design of p-SMILES with TC $>=0.400 \mathrm{~W} / \mathrm{m}-\mathrm{K}$ in details with $\alpha$ set to 100 . In Figure 5a, the probability of generated p-SMILES with predicted TC $>=0.400 \mathrm{~W} / \mathrm{m}-\mathrm{K}$ is calculated every 100 training steps. The probability of generated p-SMILES with predicted TC $>=$ $0.400 \mathrm{~W} / \mathrm{m}-\mathrm{K}$ before the training denoted as training step 0 is also calculated as a reference. A significant increase in the probability can be observed in the initial stage (from 0 to 300 steps), and then it converges to a value around 0.940 , indicating an effective reinforcement learning. Even though the generated p-SMILES with predicted TC are leveraged as feedback to train $P G_{T C}$, the generated p-SMILES are sampled randomly using $P G_{T C}$, which leads to explorative behavior during the training, thus causing some oscillation in the probability curve (more algorithmic interpretation can be found in Ref. [9, 45]). Figure 5b visualizes the predicted TC distribution of all valid generated p-SMILES every 100 training steps. The median (indicated by the orange line) 
and upper bound of the distribution are slightly oscillating during the training, which indicates the stochastic nature of such a learning algorithm. The design algorithm can exploit the area underrepresented by the training data (i.e., at the tail of the training data distribution $(0.400-0.430$ $\mathrm{W} / \mathrm{m}-\mathrm{K})$ ), but it does not generate p-SMILES with predicted TC beyond the upper bound of the training TC distribution. Learning to generate p-SMILES with high TC relies on the feedback from the regressor, which can only give a highest prediction of $0.430 \mathrm{~W} / \mathrm{m}-\mathrm{K}$ (Regressor section). One can potentially use MD simulations or experiments to evaluate the TC of generated polymers, but they are too time-consuming to label thousands of generated polymers. The current results may be improved if data for polymers with higher TC become more abundant, which should be a longterm research topic, but this does not negate our study which demonstrate a protocol to shift the generated polymer population based on the target property. Nevertheless, the fact that the reinforcement learning can significantly increase the population of polymers with $\mathrm{TC}>=0.400$ W/m-K, as shown in Figure 5c, offers useful guidance for further experimental exploration of high TC polymers.
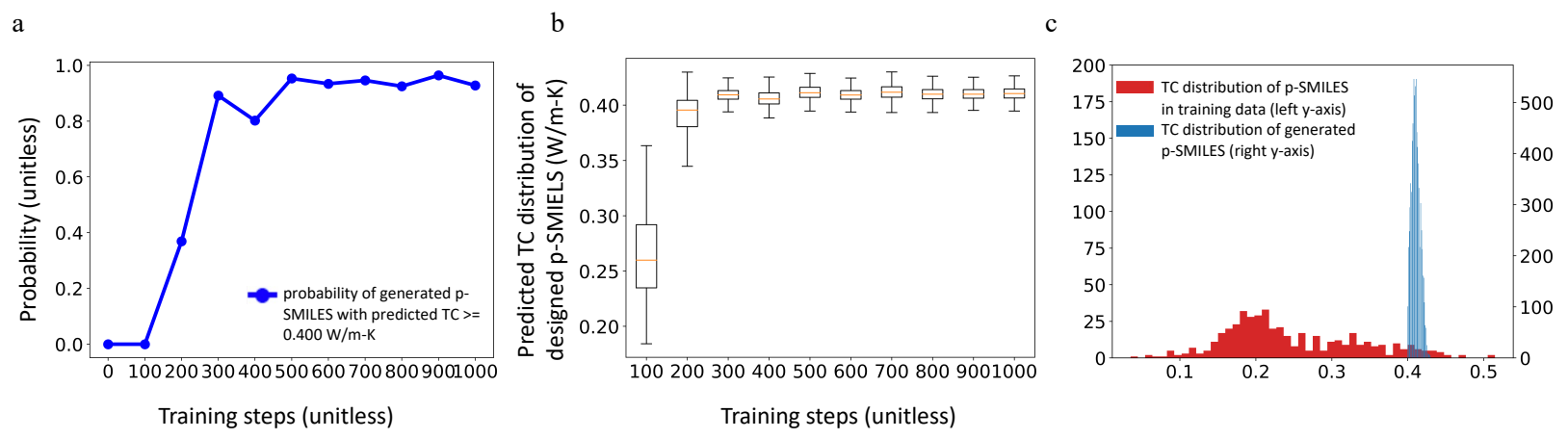

Figure 5. (a) The probability of generated p-SMILES with predicted TC $>=0.400 \mathrm{~W} / \mathrm{m}-\mathrm{K}$ at every $100^{\text {th }}$ training step as well as the $0^{\text {th }}$ training step; (b) The distribution of the predicted TC of all 
valid generated p-SMILES at every $100^{\text {th }}$ training step; (c) The TC distributions of the original 469 training data and those of the newly generated p-SMILES.

In total, we generated 7,713 p-SMILES with predicted TC greater than $0.400 \mathrm{~W} / \mathrm{m}-\mathrm{K}$. To learn the relationship between these polymers and the 12,777 real polymer from PolyInfo, the original data used to train PI1M, we visualize them in the chemical space. The p-SMILES are first represented using the 300-dimensional polymer embedding and then projected onto the 2-dimensional space for visualization using t-SNE.[44] In Figure 6a, the generated polymers cover a chemical space that is not occupied by the 12,777 real polymers from PolyInfo. Upon careful examination, there is one point at the top of the plot belongs to a real polymer from PolyInfo (highlight in a red box in Figure 6a). In Figure 6b, each polymer in the 2-dimensional t-SNE space is labeled by the regressor-predicted TC values with a color scale, and it is seen that the solo PolyInfo point at the top of the t-SNE plot has a TC $>0.400 \mathrm{~W} / \mathrm{m}-\mathrm{K}$. It is interesting to see how reinforcement learning was able to utilize a single point with high TC to populate the chemical space with high TC though almost no training data covers the same space. We further decompose the 7,713 generated polymers into different subgroups, based on the training steps when they are generated. We first analyze the generated p-SMILES between the $100^{\text {th }}$ and the $300^{\text {th }}$ training step in Figure $\mathbf{6 c}$, where significant learning happens (see Figure 5a). The algorithm first tries to find those desired polymers sparsely distributed outside of the space covered by the PolyInfo polymers (see points for the $200^{\text {th }}$ training step), and then densifies the population around them (based on the data for the $300^{\text {th }}$ training step). Such graduality indicates a continuous learning nature in polymer design based on reinforcement learning. As the learning process converges (from the $400^{\text {th }}$ to the $1000^{\text {th }}$ training step, see Figure 6d), the generated polymers gradually fill the space not covered by the 
previously generated polymers, without extending significantly into new space. This may again be attribute to the limitation of the regressor which is only effective to predict TC as high as 0.430 $\mathrm{W} / \mathrm{m}-\mathrm{K}$, as discussed in the last paragraph.
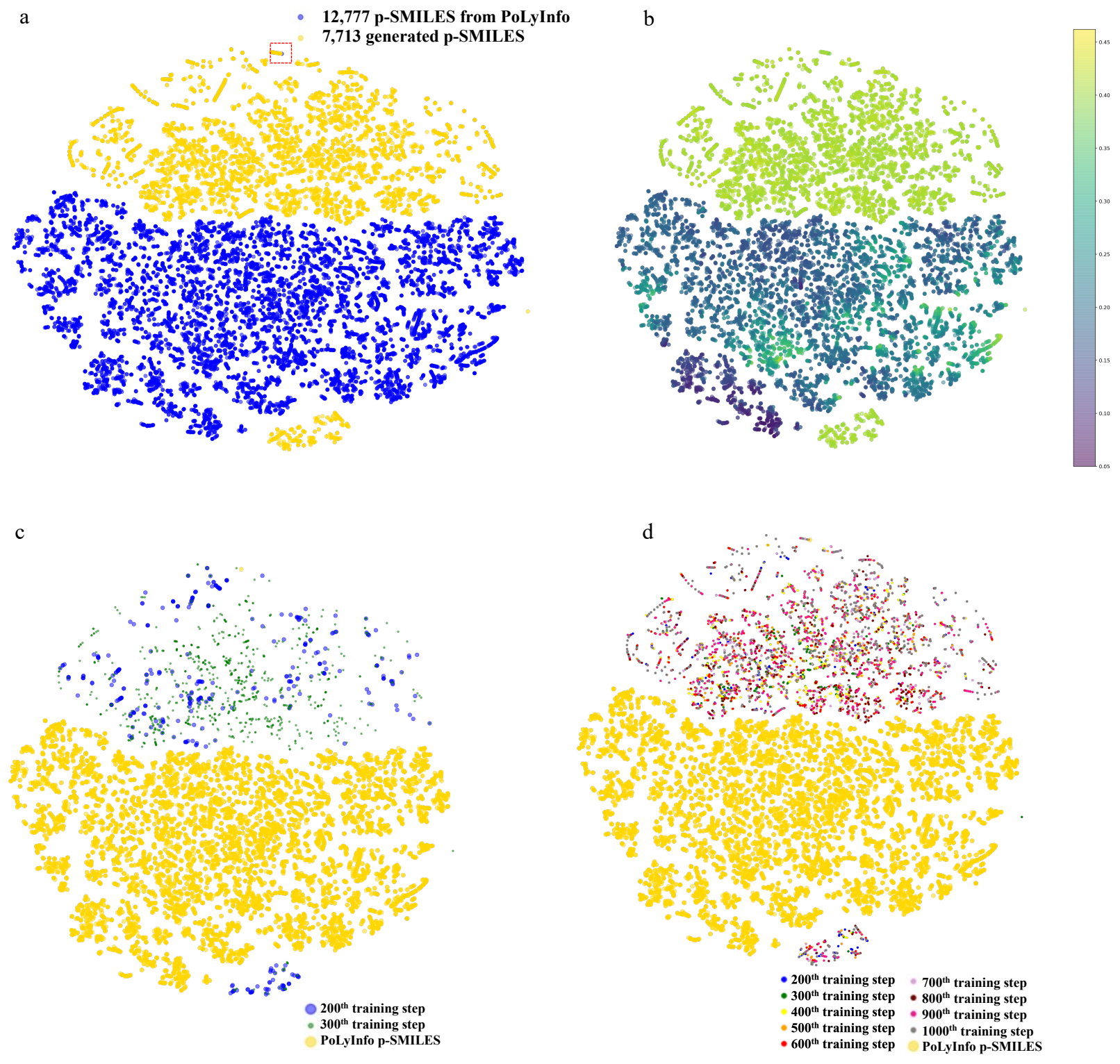

Figure 6. (a) The visualization of 7,713 generated polymers and 12,777 real polymers from PolyInfo using t-SNE; (b) the visualization of these polymers with their predicted TC labeled in 
color scale; (c) the visualization of the generated polymers in the $200^{\text {th }}$ and the $300^{\text {th }}$ training step, as well as the 12,777 real polymers from PoLyInfo; (d) the visualization of the 7,713 generated divided into different subgroups based on the training step when they are generated.

After acquiring the generated p-SMILES with predicted TC $>=0.400 \mathrm{~W} / \mathrm{m}-\mathrm{K}$, we validate their TC using MD simulation, which was used for creating the training TC data. The simulation details are the same with those described in Ref. [31]. There is a total of 7,713 generated p-SMIELS with TC $>=0.400 \mathrm{~W} / \mathrm{m}-\mathrm{K}$ but validating all of them is insurmountable. Thus, criteria for selecting generated polymers for validation are needed. We do not use the predicted TC value as a criterion as long as they are above $0.400 \mathrm{~W} / \mathrm{m}-\mathrm{K}$, because the MD-calculated TC has a uncertainty of $\sim 20 \%$ according to the previous study.[31] A perceived challenge that may obstruct the usefulness of a molecular generator is that the proposed molecules may be synthetically inaccessible.[46] Considering that the ultimate goal is to synthesize the proposed p-SMILES for real-world applications in the future, we introduce the synthetic accessibility (SA) score as one criterion for selecting p-SMILES. The Schuffenhauer's SA score[47] (SA score for short) is frequently employed to evaluate the synthesizability of molecules, which is a heuristic approach developed to estimate how hard (10.000) or how easy (1.000) it may be to synthesize a given molecule.

To identify an appropriate SA score threshold, we collect 12,777 p-SMILES that correspond to existing homopolymers from the PolyInfo[5] database and estimate their synthesizability using the SA score. We note that the polymers contained in the PolyInfo database are all experimentally synthesized polymers. Based on the results in Figure 7a, 11,555 out of 12,777 p-SMILES (or $90.436 \%$ ) have SA score $<=5.00$, and the lowest and highest values are respectively 2.454 and 
8.201. Thus, a SA score of 5.00 should indicate a synthesizable polymer with reasonable chemical reaction processes. We then calculate the SA score for our 7,713 generated p-SMILES, and the distribution is shown in Figure 7b. The 7,713 generated p-SMILES all have SA scores below 5.044 , with $99.974 \%$ of them (or 7,711 out of 7,713) below 5.000. We use SA score $<5.000$ as a criterion for selecting candidate p-SMILES for further validation of the TC using MD simulations.

A well-known limitation of MD simulation is the availability of force field parameters. The generated p-SMILES, even after passing the synthesizability criterion, are not guaranteed to be successfully simulatable in MD simulations. Thus, we first pass the 7,711 generated p-SMILES with SA score below 5.000 into the amorphous polymer structure generation pipeline developed in Ref. [31], to obtain their initial amorphous structures (before systematic optimization process) that can be potentially leveraged for TC calculations later. We identify 2,674 p-SMILES that can have their initial amorphous structures constructed, while the failure of the rest is due to the lack of force field parameters available in the MD simulation pipeline, which uses GAFF2 (General AMBER Force Field 2).[48]

An additional criterion that can be implemented is the chemical diversity of the designed pSMILES. According to Ref. [31], in our training data, 35 p-SMILES with TC $>=0.400 \mathrm{~W} / \mathrm{m}-\mathrm{K}$ come from 7 polymer classes, whose $\mathrm{TC}$ values range from 0.400 to $0.515 \mathrm{~W} / \mathrm{m}-\mathrm{K}$. The variation in TC of the 35 p-SMILES is larger than that from any single polymer class, demonstrating that diversifying the p-SMILES might lead to a wider TC range, which can potentially benefit the successfully identification of polymers with higher TC. To select diverse p-SMILES for further validation, we first cluster 2,674 p-SMILES that can have their initial amorphous structures 
generated into five groups based on the KMeans clustering algorithm (Figure 7c),[49] where the p-SMILES are first represented by polymer embeddings (300-dimensional vectors), and then compressed onto the 2-dimensional space via t-SNE,[44] which are further clustered by KMeans implemented using scikit-learn.[37] We then select 10 polymers that are the nearest to each cluster center (positions indicated by the red "x" in Figure 7c) for each cluster. Among the total of $50 \mathrm{p}-$ SMILES, 30 of them can go through the complete TC calculation using the MD simulation pipeline developed in Ref. [31], with the rest failed to pass through the amorphous structural optimization mostly due to inappropriate force field parameters.
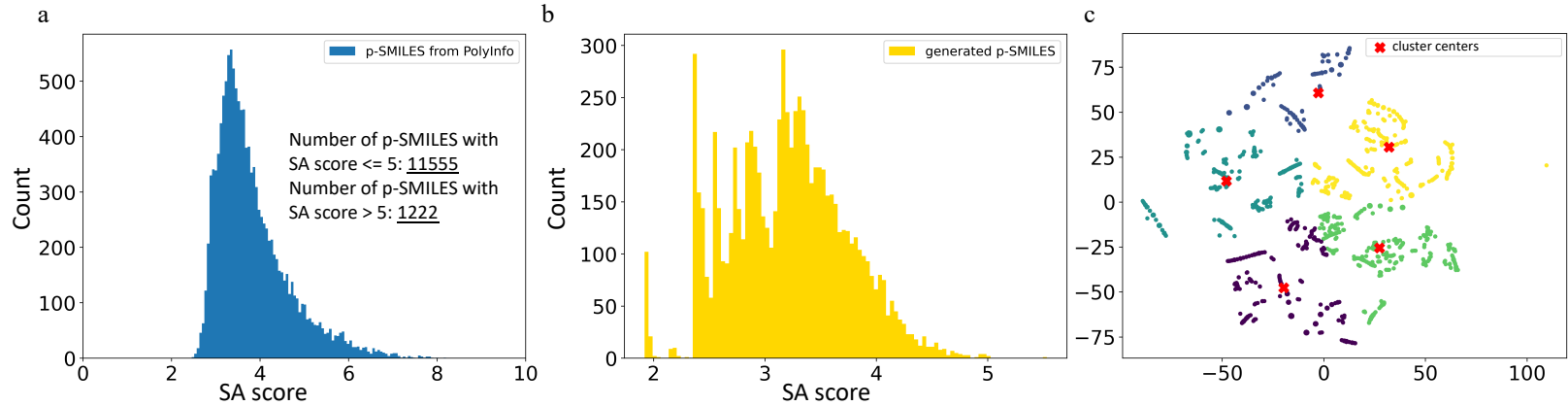

Figure 7. (a) The SA score distribution of all the 12,777 p-SMILES from the PolyInfo database that are existing. 11,555 of them have SA score below 5.000, and 1,222 have SA score above 5.000; (b) The SA score distribution of the 7,713 generated p-SMILES with TC $>0.400 \mathrm{~W} / \mathrm{m}-\mathrm{K}$; (c) The visualization of the 2,674 p-SMILES that can be simulated using MD in a 2D chemical space (the $\mathrm{x}$-axis and $\mathrm{y}$-axis are unitless), where the p-SMILES are clustered into five groups based on KMeans, and each color represents a group. The red crosses are the cluster centers. 
The MD-calculated TC values of the 30 polymers are shown in Table 2. Among these polymers, a diversity in chemical elements as well as monomer length can be observed. These calculated polymers are also indicated in Figure 7c, which indicate their diversity in the chemical space. We note that as indicated in our previous study,[31] the ensemble difference due randomness in initial amorphous polymer packing conformation presents the largest uncertainty in the calculated TC. The reported TC values in Table 2 are averaged over three independent ensembles for each polymer and the error is the standard deviation. The TC of these 30 p-SMILES range from 0.360 $\mathrm{W} / \mathrm{m}-\mathrm{K}$ to $0.693 \mathrm{~W} / \mathrm{m}-\mathrm{K} .25$ out of the 30 are validated to have TC greater than the target 0.400 $\mathrm{W} / \mathrm{m}-\mathrm{K}$, and all of them are found to have TC $>0.360 \mathrm{~W} / \mathrm{m}-\mathrm{K}$. Considering the uncertainty of the MD-calculated TC $(\sim 10 \%)$, and the fact that the RF regressor used may not be very precise much beyond $\mathrm{TC}=0.400 \mathrm{~W} / \mathrm{m}-\mathrm{K}$ due to the training data range, we deem the MD-validation results reasonable.

Table 2. The MD-calculated TC for selected generated polymers, their predicted TC from the RF regressor and SA scores. All corresponding monomer structures are shown in the Appendix.

\begin{tabular}{|c|c|c|c|c|}
\hline & p-SMILES & SA score & Predicted TC $(\mathrm{W} / \mathrm{m}-\mathrm{K})$ & MD-labeled TC (W/m-K) \\
\hline 1 & ${ }^{*}$ CCCCCCCNCCCCCCCCCCCCCCCCCCCCCCCCCCCCCCCN* & 2.549 & 0.412 & $0.546 \pm 0.038$ \\
\hline 2 & */CCCCCCCCNCCCCCCCCCCCCCCCCCCCCCCCCCCCCCCN* & 2.549 & 0.4 & $0.464 \pm 0.043$ \\
\hline 3 & ${ }^{*}$ CCCCCCCCCCCCCCCCCCCCNCCCCCCCCCCCCCCCCCCN* & 2.549 & 0.4 & $0.602 \pm 0.031$ \\
\hline 4 & ${ }^{*}$ CCCCCCCCCCCCCCCCCCCCCCCCNCCCCCCCCCCCCCCN* & 2.549 & 0.401 & $0.485 \pm 0.039$ \\
\hline 5 & ${ }^{*} \mathrm{CCCCCCCC} / \mathrm{CCCCCCCCCCCCCCCCCCCCCCCCCNCCCN}{ }^{*}$ & 2.591 & 0.401 & $0.693 \pm 0.044$ \\
\hline 6 & ${ }^{*} \mathrm{CCCCC} / \mathrm{CCCCCCCCCCCCCCCNCCCCCCCCCCCCCCN}{ }^{*}$ & 2.549 & 0.402 & $0.564 \pm 0.039$ \\
\hline 7 & ${ }^{*}$ CCCCCCCCCCCCCCCCCCCCCCCCNCCCC/CCCCCCCCCCN* & 2.549 & 0.402 & $0.445 \pm 0.030$ \\
\hline 8 & ${ }^{*}$ CCCCCCCCCCCCCCCNCCCCCCCCCCCCCCCCCCCCCCCN $*$ & 2.549 & 0.402 & $0.644 \pm 0.038$ \\
\hline 9 & ${ }^{*}$ C/CCCCCCCCCCCC/CCCCCCCCCCCCCCCCCCCCNCCCCCN ${ }^{*}$ & 2.549 & 0.402 & $0.561 \pm 0.048$ \\
\hline 10 & ${ }^{*}$ CCCCCCCC/CCCCCCCCCCCCCCCCC/CCCCCCNCCCCCCCN $*$ & 2.549 & 0.403 & $0.494 \pm 0.025$ \\
\hline 11 & ${ }^{*} \mathrm{CCCCC/CСССССССССССССССССССССCSS} *$ & 2.61 & 0.403 & $0.404 \pm 0.038$ \\
\hline
\end{tabular}




\begin{tabular}{|c|c|c|c|c|}
\hline 12 & ${ }^{*}$ C/CCCCCCCCCCCCCCCCCCCCCCCCCCCCCCSS ${ }^{*}$ & 2.595 & 0.403 & $0.381 \pm 0.025$ \\
\hline 13 & ${ }^{*}$ CCCCCCCCCCCCCCCCCCCCCCCCCCCCCCSS* & 2.582 & 0.405 & $0.367 \pm 0.034$ \\
\hline 14 & $\begin{array}{c}{ }^{*} \text { CCCCCCCCCCCCCCCCCCCCCCCCCCCCCCCCCCCCCCCCC } \\
\text { CSS }{ }^{*}\end{array}$ & 2.556 & 0.409 & $0.425 \pm 0.033$ \\
\hline 15 & $\begin{array}{c}{ }^{*} \mathrm{CCCCCCCCCCCCCCCCCCCCCCCCCCCCCCSCCCCCCCCC} \\
\mathrm{CCCCC} / \mathrm{CCC} / *\end{array}$ & 2.484 & 0.41 & $0.484 \pm 0.021$ \\
\hline 16 & $\begin{array}{c}{ }^{*} \text { CCCCCCCCCCCCCCCCCCCCCCCCCCCCCCCCCCSCCCCC } \\
\text { СCCCCCC }{ }^{*}\end{array}$ & 2.475 & 0.411 & $0.544 \pm 0.028$ \\
\hline 17 & $\begin{array}{c}{ }^{*} \mathrm{CCCCCCC} / \mathrm{CCCCC} / \mathrm{CCCCCCCCCCCCC/CCCCCCCCCCCCCC} \\
\mathrm{CCS}^{*}\end{array}$ & 2.403 & 0.411 & $0.635 \pm 0.033$ \\
\hline 18 & $\begin{array}{c}* / \mathrm{CCCCCCCCCCC} / \mathrm{CCCCCCCCCCCCCCCCCCCCCCCCCCC} \\
\mathrm{CS}^{*}\end{array}$ & 2.398 & 0.412 & $0.428 \pm 0.040$ \\
\hline 19 & ${ }^{*}$ CCCCCCCCCCCCCCC/CCCCCCCCCCCCCCCCCCCCS* & 2.393 & 0.412 & $0.529 \pm 0.019$ \\
\hline 20 & *SCCCCCCCCCCCCCCCCCCCCCCCCCCCCCCCCCCCC* & 2.397 & 0.412 & $0.416 \pm 0.030$ \\
\hline 21 & ${ }^{*} \mathrm{CCCCCCCCCCCCCCCCNCCCCCCCSCCCCCCCCN} *$ & 2.923 & 0.412 & $0.426 \pm 0.029$ \\
\hline 22 & ${ }^{*}$ CCCCCCCCCCC/SCCCCCNCCCCCCCCCCCCCCCN* & 2.923 & 0.412 & $0.399 \pm 0.026$ \\
\hline 23 & ${ }^{*}$ CCCCCCCCCCCCCCSCCCCCCCCCCCCCCCNCCCCN ${ }^{*}$ & 2.901 & 0.412 & $0.411 \pm 0.029$ \\
\hline 24 & ${ }^{*}$ CCCCCCCCCCCCCCCCCCCNCCCCCCCCCCCCSCCCN* & 2.927 & 0.412 & $0.413 \pm 0.039$ \\
\hline 25 & ${ }^{*}$ CCCCCCCCCCCCCCCCCNCCCCCCCCCCCCSCCCCCN ${ }^{*}$ & 2.901 & 0.412 & $0.360 \pm 0.024$ \\
\hline 26 & $\begin{array}{c}{ }^{*} \mathrm{CCNCCCCCCCCCCCCCCCCCNCCCCCCCCCCCCCCCCSCC} \\
\mathrm{CCN}^{*}\end{array}$ & 2.921 & 0.412 & $0.399 \pm 0.031$ \\
\hline 27 & $\begin{array}{c}{ }^{*} \mathrm{CCCCCCCCCCCCCCCCCCCCCCSCCCCCCCCCNCCCCCCN} \\
\mathrm{CCCCN}^{*}\end{array}$ & 2.941 & 0.412 & $0.439 \pm 0.028$ \\
\hline 28 & $\begin{array}{c}{ }^{*} \mathrm{NCCCNCCCCCCCCCCNCCCCCCCCCCCCCCCCCCCCCSCC} \\
\text { CCCCCC* }\end{array}$ & 2.978 & 0.415 & $0.493 \pm 0.036$ \\
\hline 29 & $\begin{array}{c}{ }^{*} \mathrm{CCCCCCCCCCCCCCCCCSCCCCCCCCCCCCCCNCCCCCNC} \\
\text { CCCCCCN } *\end{array}$ & 2.952 & 0.416 & $0.463 \pm 0.035$ \\
\hline 30 & ${ }^{*}$ CCCCCCCCCCCCCCCCCCCCCCNCCCCCCCCCCSCCCN ${ }^{*}$ & 2.919 & 0.419 & $0.420 \pm 0.038$ \\
\hline
\end{tabular}

\section{CONCLUSION}

In summary, we successfully designed a large number of polymers with high TC based on reinforcement learning and MD-labeled TC data for 469 polymers from Ref. [31]. We have also used MD simulation to verify that 25 out of 30 validated polymers indeed have TC $>0.400 \mathrm{~W} / \mathrm{m}$ $\mathrm{K}$, which was the threshold in the design task. We also discussed the process of design in details 
based on hyperparameter study and statistical analysis of the generated polymers. The reinforcement learning-based inverse design is shown to effectively shift the distribution of polymers towards those with high TC. By anchoring the polymer generator to a benchmark distribution through formulating an appropriate loss function, we have achieved high fraction in the generated polymers that are chemically valid. To make the generated polymers potentially useful for real-world applications, we also evaluated their synthetic accessibility, and most of them are found to be readily synthesizable. Besides synthetic accessibility score, the selected polymers for MD validation are chosen to be chemically diverse based on a clustering algorithm. The best thermally conductive polymer validated has a TC of $0.693 \mathrm{~W} / \mathrm{m}-\mathrm{K}$, which is also estimated to be easily synthesized. Our work may set a useful example for the inverse design of polymers given target properties to achieve polymer-by-design.

\section{ACKNOWLEDGEMENT}

The computation is supported in part by the University of Notre Dame, Center for Research Computing, and NSF through XSEDE resources provided by TACC Stampede II under a grant number TG-CTS100078.

\section{APPENDIX}

1. *CCCCCCCNCCCCCCCCCCCCCCCCCCCCCCCCCCCCCCCN*

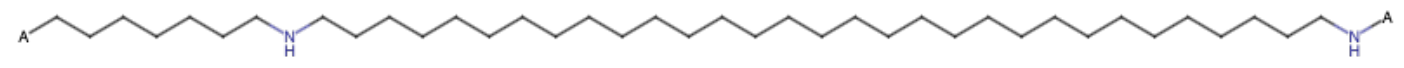

2. */CCCCCCCCNCCCCCCCCCCCCCCCCCCCCCCCCCCCCCCN*

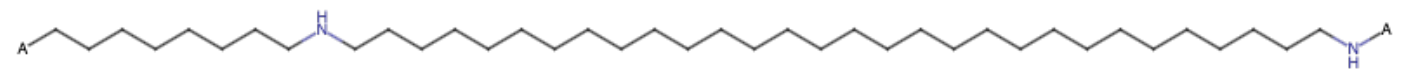

\section{3. *CCCCCCCCCCCCCCCCCCCCNCCCCCCCCCCCCCCCCCCN*}


(C)

4. ${ }^{*} \mathrm{CCCCCCCCCCCCCCCCCCCCCCCCNCCCCCCCCCCCCCCN*}$

(C)

5. * $\mathrm{CCCCCCCC} / \mathrm{CCCCCCCCCCCCCCCCCCCCCCCCCCCNCCCN} *$

准

6. ${ }^{*} \mathrm{CCCCC} / \mathrm{CCCCCCCCCCCCCCCCCNCCCCCCCCCCCCCCCCN} *$

(N)

7. ${ }^{*} \mathrm{CCCCCCCCCCCCCCCCCCCCCCCCNCCCC/CCCCCCCCCCN} *$

(1)

8. *CCCCCCCCCCCCCCCNCCCCCCCCCCCCCCCCCCCCCCCN*

氺

9. ${ }^{*} \mathrm{C} / \mathrm{CCCCCCCCCCCC} / \mathrm{CCCCCCCCCCCCCCCCCCCCNCCCCCN} *$

(N)

10. * $\mathrm{CCCCCCCC/CCCCCCCCCCCCCCCCC/CCCCCCNCCCCCCCN*}$

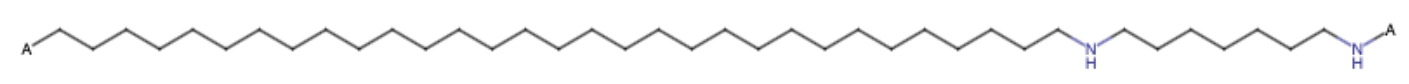

11. *CCCCC/CCCCCCCCCCCCCCCCCCCCCCCCCSS*

等

12. * $\mathrm{C} / \mathrm{CCCCCCCCCCCCCCCCCCCCCCCCCCCCCCSS*}$

(N)

13. ${ }^{*} \mathrm{CCCCCCCCCCCCCCCCCCCCCCCCCCCCCCCCSS} *$ 
(N)

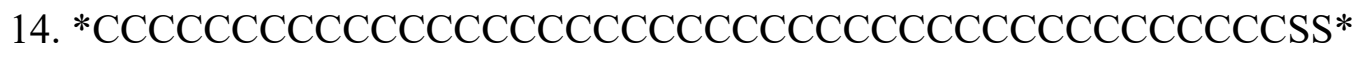

(1)

15. ${ }^{*} \mathrm{CCCCCCCCCCCCCCCCCCCCCCCCCCCCCCCSCCCCCCCCCCCCCC/CCC/*}$

(N)

16. * ${ }^{* C C C C C C C C C C C C C C C C C C C C C C C C C C C C C C C C C C C S C C C C C C C C C C C C * ~}$

䍩

17. ${ }^{*} \mathrm{CCCCCCC} / \mathrm{CCCCC} / \mathrm{CCCCCCCCCCCCCC/CCCCCCCCCCCCCCCCS*}$

(A)

18. */CCCCCCCCCCCC/CCCCCCCCCCCCCCCCCCCCCCCCCCCCCCS*

(1)

19. ${ }^{*} \mathrm{CCCCCCCCCCCCCCCC/CCCCCCCCCCCCCCCCCCCCCS*}$

(3)

20. *SCCCCCCCCCCCCCCCCCCCCCCCCCCCCCCCCCCCC*

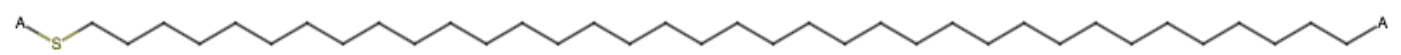

21. * ${ }^{* C C C C C C C C C C C C C C C C N C C C C C C C S C C C C C C C C N *}$<smiles>CCCCCCCCCCCCCCCCCCCCCNCCCCCCCCCCCCCCCCCC</smiles>

22. ${ }^{*} \mathrm{CCCCCCCCCCC} / \mathrm{SCCCCCNCCCCCCCCCCCCCCCCN} *$

(N)

23. *CCCCCCCCCCCCCCSCCCCCCCCCCCCCCCCNCCCCN* 
舟

24. *CCCCCCCCCCCCCCCCCCCNCCCCCCCCCCCCSCCCN*

(N)

25. ${ }^{*} \mathrm{CCCCCCCCCCCCCCCCCNCCCCCCCCCCCCSCCCCCN*}$

(N)

26. *CCNCCCCCCCCCCCCCCCCCCNCCCCCCCCCCCCCCCCCSCCCCN*

(N)

27. *CCCCCCCCCCCCCCCCCCCCCCCSCCCCCCCCCCNCCCCCCNCCCCN*

"

28. *NCCCNCCCCCCCCCCCNCCCCCCCCCCCCCCCCCCCCCCSCCCCCCCC*

(1)

29. *CCCCCCCCCCCCCCCCCCSCCCCCCCCCCCCCCCNCCCCCNCCCCCCCN*

$\mathrm{NON}^{2}$

30. *CCCCCCCCCCCCCCCCCCCCCNCCCCCCCCCCSCCCN*

(N) 


\section{Reference}

1. Silver, D., et al., Mastering the game of Go without human knowledge. Nature, 2017. 550(7676): p. 354-359.

2. Senior, A.W., et al., Improved protein structure prediction using potentials from deep learning. Nature, 2020. 577(7792): p. 706-710.

3. He, K., Zhang, X., Ren, S. and Sun, J., Deep residual learning for image recognition. In Proceedings of the IEEE conference on computer vision and pattern recognition, 2016: p. 770-778.

4. Irwin, J.J., et al, ZINC: a free tool to discover chemistry for biology. Journal of chemical information and modeling, 2012. 52(7): p. 1757-1768.

5. Otsuka, S., et al, PoLyInfo: Polymer database for polymeric materials design. Emerging Intelligent Data and Web Technologies (EIDWT), 2011 International Conference on. IEEE, 2011.

6. Kim, C., et al., Polymer Genome: A Data-Powered Polymer Informatics Platform for Property Predictions. The Journal of Physical Chemistry C, 2018. 122(31): p. 1757517585.

7. Jain, A., Ong, S.P., Hautier, G., Chen, W., Richards, W.D., Dacek, S., Cholia, S., Gunter, D., Skinner, D., Ceder, G. and Persson, K.A., Commentary: The Materials Project: A materials genome approach to accelerating materials innovation. APL materials, 2013. 1(1).

8. Popova, M., Olexandr Isayev, and Alexander Tropsha, Deep reinforcement learning for de novo drug design. Science advances, 2018. 4(7): p. eaap7885.

9. Olivecrona, M., et al., Molecular de-novo design through deep reinforcement learning. $\mathrm{J}$ Cheminform, 2017. 9(1): p. 48.

10. Gomez-Bombarelli, R., et al., Automatic Chemical Design Using a Data-Driven Continuous Representation of Molecules. ACS Cent Sci, 2018. 4(2): p. 268-276.

11. Griffiths, R.-R., and José Miguel Hernández-Lobato, Constrained Bayesian optimization for automatic chemical design using variational autoencoders. Chemical Science, 2020.

12. Mao, Y., He, Q. and Zhao, X., Designing complex architectured materials with generative adversarial networks. Science Advances, 2020. 6(17): p. eaaz4169.

13. Wu, S., et al., Machine-learning-assisted discovery of polymers with high thermal conductivity using a molecular design algorithm. npj Computational Materials, 2019. 5(1): p. 1-11.

14. Henry, A., Thermal transport in polymers. Annual Review of Heat Transfer, 2014. 17.

15. Mehra, N., et al., Thermal transport in polymeric materials and across composite interfaces. Applied Materials Today, 2018. 12: p. 92-130.

16. $\mathrm{Xu}, \mathrm{X}$., Chen, J., Zhou, J. and Li, B., Thermal conductivity of polymers and their nanocomposites. Advanced Materials, 2018. 30(17).

17. Wei, X., Wang, Z., Tian, Z. and Luo, T., Thermal Transport in Polymers: A Review. arXiv preprint arXiv, 2020. 2009(09430).

18. Zhang, T. and T. Luo, Role of Chain Morphology and Stiffness in Thermal Conductivity of Amorphous Polymers. J Phys Chem B, 2016. 120(4): p. 803-12.

19. Wei, X., T. Zhang, and T. Luo, Chain conformation-dependent thermal conductivity of amorphous polymer blends: the impact of inter-and intra-chain interactions. Phys Chem Chem Phys, 2016. 18(47): p. 32146-32154. 
20. Wei, X. and T. Luo, Role of Ionization in Thermal Transport of Solid Polyelectrolytes. The Journal of Physical Chemistry C, 2019.

21. Wei, X., R. Ma, and T. Luo, Thermal Conductivity of Polyelectrolytes with Different Counterions. The Journal of Physical Chemistry C, 2020. 124(8): p. 4483-4488.

22. Shanker, A., Li, C., Kim, G.H., Gidley, D., Pipe, K.P. and Kim, J., High thermal conductivity in electrostatically engineered amorphous polymers. Science advances, 2017. 3(7).

23. Kim, G.H., et al., High thermal conductivity in amorphous polymer blends by engineered interchain interactions. Nat Mater, 2015. 14(3): p. 295-300.

24. Lu, T., Kim, K., Li, X., Zhou, J., Chen, G. and Liu, J., Thermal transport in semicrystalline polyethylene by molecular dynamics simulation. Journal of Applied Physics, 2018. 123(1).

25. Pan, S.J., and Qiang Yang, A survey on transfer learning. IEEE Transactions on knowledge and data engineering, 2009. 22(10): p. 1345-1359.

26. Gong, Z., et al., Predicting Thermodynamic Properties of Alkanes by High-Throughput Force Field Simulation and Machine Learning. J Chem Inf Model, 2018. 58(12): p. 25022516.

27. Tao, L., G. Chen, and Y. Li, Machine learning discovery of high-temperature polymers. Patterns (N Y), 2021. 2(4): p. 100225.

28. Mannodi-Kanakkithodi, A., Pilania, G., Huan, T.D., Lookman, T. and Ramprasad, R., Machine learning strategy for accelerated design of polymer dielectrics. Scientific reports, 2016. 6(1): p. 1-10.

29. Doan Tran, H., Kim, C., Chen, L., Chandrasekaran, A., Batra, R., Venkatram, S., Kamal, D., Lightstone, J.P., Gurnani, R., Shetty, P. and Ramprasad, M., Machine-learning predictions of polymer properties with Polymer Genome. Journal of Applied Physics, 2020. 128(17).

30. Palizhati, A., et al., Toward Predicting Intermetallics Surface Properties with HighThroughput DFT and Convolutional Neural Networks. J Chem Inf Model, 2019. 59(11): p. 4742-4749.

31. Ma, R., Zhang, H., Xu, J., Hayashi, Y., Yoshida, R., Shiomi, J. and Luo, T., Machine Learning-Assisted Exploration of Thermally Conductive Polymers Based on HighThroughput Molecular Dynamics Simulations. arXiv preprint arXiv, 2021. 2109(02794).

32. Ma, R. and T. Luo, PIIM: A Benchmark Database for Polymer Informatics. J Chem Inf Model, 2020. 60(10): p. 4684-4690.

33. Weininger, D., SMILES, a chemical language and information system. 1. Introduction to methodology and encoding rules. Journal of chemical information and computer sciences, 1988. 28(1): p. 31-36.

34. Khalifeh, S., Polymers in Organic Electronics: Polymer Selection for Electronic, Mechatronic, and Optoelectronic Systems. Elsevier, 2020.

35. Kang, S. and K. Cho, Conditional Molecular Design with Deep Generative Models. J Chem Inf Model, 2018.

36. Breiman, L., Random forests. Machine Learning, 2001. 45(1): p. 5-32.

37. Pedregosa, F., et al, Scikit-learn: Machine learning in Python. Journal of machine learning research, 2011. 12(10): p. 2825-2830.

38. Schuster, M., and Kuldip K. Paliwal, Bidirectional recurrent neural networks. IEEE Transactions on Signal Processing, 1997. 45(11): p. 2673-2681. 
39. Werbos, P.J., Backpropagation through time: what it does and how to do it. Proceedings of the IEEE, 1990. 78(10): p. 1550-1560.

40. Chung, J., Gulcehre, C., Cho, K. and Bengio, Y., Empirical evaluation of gated recurrent neural networks on sequence modeling. arXiv preprint arXiv, 2014. 1412(3555).

41. Kingma, D.P., and Jimmy Ba, Adam: A method for stochastic optimization. arXiv preprint arXiv, 2014. 1412(6980).

42. Paszke, A., et al., PyTorch: An imperative style, high-performance deep learning library. Advances in Neural Information Processing Systems, 2019.

43. Landrum, G., RDKit: Open-Source Cheminformatics Software.(2016). URL http://www.rdkit.org/, https://github.com/rdkit/rdkit, 2016.

44. Maaten, L.v.d., and Geoffrey Hinton, Visualizing data using t-SNE. Journal of machine learning research, 9.Nov 2008: p. 2579-2605.

45. Schulman, J., Wolski, F., Dhariwal, P., Radford, A. and Klimov, O., Proximal policy optimization algorithms. arXiv preprint arXiv, 2017. 1707(06347).

46. Gao, W. and C.W. Coley, The Synthesizability of Molecules Proposed by Generative Models. J Chem Inf Model, 2020. 60(12): p. 5714-5723.

47. Ertl, P., and Ansgar Schuffenhauer, Estimation of synthetic accessibility score of drug-like molecules based on molecular complexity and fragment contributions. Journal of cheminformatics, 2009. 1(1).

48. Vassetti, D., Marco Pagliai, and Piero Procacci, Assessment of GAFF2 and OPLS-AA General Force Fields in Combination with the Water Models TIP3P, SPCE, and OPC3 for the Solvation Free Energy of Druglike Organic Molecules. Journal of chemical theory and computation, 2019. 15(3): p. 1983-1995.

49. Krishna, K.a.M., M.N., Genetic K-means algorithm. IEEE Transactions on Systems, Man, and Cybernetics, Part B (Cybernetics), 1999. 29(3): p. 433-439. 